\title{
Acadêmicos de Odontologia no combate à COVID-19: percepção discente sobre um projeto de extensão interprofissional
}

\author{
Thabata Cristy Zermiani*; Ruann Oswaldo Carvalho da Silva**; Rubia Daniela Thieme***; Erildo \\ Vicente Muller****; Rafael Gomes Ditterich*****
}

* Doutora em Políticas Públicas, Professora assistente, Curso de Odontologia, Pontifícia Universidade Católica do Paraná

** Doutorando em Políticas Públicas na Universidade Federal do Paraná e em Odontologia na Pontifícia Universidade Católica do Paraná

*** Doutoranda em Políticas Públicas, Professora Assistente, Curso de Nutrição, Universidade Positivo

**** Doutor em Saúde Coletiva, Professor associado, Universidade Estadual de Ponta Grossa

***** Doutor em Odontologia (Saúde Coletiva), Professor Associado, Curso de Odontologia, Universidade Federal do Paraná

Recebido: 28/06/2021. Aprovado: 30/11/2021.

\begin{abstract}
RESUMO
Este artigo tem como objetivo relatar a percepção dos discentes de Odontologia quanto sua participação nas ações extensionistas interprofissionais no enfrentamento da pandemia de COVID19. Para tanto, foi realizado um estudo qualitativo, transversal e descritivo, por meio de roteiro com perguntas abertas e questionário estruturado autoaplicado, enviado para estudantes participantes no Projeto de Extensão Universitária por meio da atenção às divisas rodoviárias do Estado do Paraná e/ou da atuação como atendentes em serviço de telessaúde para enfrentamento da COVID-19 (Central de Informações COVID-19) e/ou na Farmácia Especial do Estado do Paraná. A análise dos relatos foi realizada por meio do Discurso do Sujeito Coletivo. Todos os estudantes de Odontologia que participaram do projeto aceitaram responder a essa pesquisa. Foram identificadas como principais contribuições do Projeto de Extensão para a sua formação a obtenção de novos conhecimentos, a oportunidade de atuação interprofissional, a aplicação prática dos conhecimentos teóricos, o aprendizado técnico-científico, a promoção de acolhimento, da escuta qualificada e de atendimento humanizado. Deste modo, conclui-se que está atendendo às diretrizes para a Extensão na Educação Superior Brasileira, promovendo a formação integral e cidadã dos estudantes, propiciando a vivência dos seus conhecimentos, de modo interprofissional e interdisciplinar.

Descritores: Infecções por Coronavírus. Odontologia. Relações Comunidade-Instituição. Educação.
\end{abstract}




\section{INTRODUÇÃO}

De acordo com as Diretrizes Curriculares do Curso de Odontologia ${ }^{1}$ de 2021, deve-se incluir o Sistema Único de Saúde (SUS) como etapa integrante na formação dos cirurgiões-dentistas, com ênfase na atenção integral à saúde, e na formação de profissionais promotores da saúde e aptos para a atuação em equipe interprofissional, interdisciplinar e transdisciplinar.

Todo ofício é uma devolutiva à sociedade, em que o profissional precisa ser e estar preparado para exercer a função técnico-social do seu trabalho. Diante desse cenário é fundamental a formação mais holística e humana dentro da academia, com a aquisição de conhecimentos contribuindo para o desenvolvimento de ideias, facilitando a construção de autonomia, e promovendo a formação de cidadãos e trabalhadores conscientes ${ }^{2-3}$.

Neste sentido, destaca-se o papel da Extensão Universitária, a qual é definida pela Resolução $\mathrm{n}^{\circ} 7$ de 18 de dezembro de $2018^{4}$, como "[...] a atividade que se integra à matriz curricular $\mathrm{e}$ à organização da pesquisa, constituindo-se em processo interdisciplinar, político educacional, cultural, científico, tecnológico, que promove a interação transformadora entre as instituições de ensino superior e os outros setores da sociedade, por meio da produção e da aplicação do conhecimento, em articulação permanente com o ensino e a pesquisa". Assim, proporciona aos estudantes formação cidadã integrada à matriz curricular ${ }^{4}$, permitindo socializar o conhecimento e promover o diálogo entre o saber científico e o saber popular ${ }^{1,3,5}$.

Em dezembro de 2019, inúmeros casos de uma desconhecida infecção respiratória foram reportados em Wuhan, China, associados à exposição de indivíduos em um mercado de comercialização de animais ${ }^{6}$. Descobriu-se, então, que se tratavam dos primeiros casos do novo coronavírus denominado de Coronavírus da
Síndrome Respiratória Aguda Grave - 2 (SARSCoV-2), o qual é causador do que foi denominado de doença do novo Coronavírus (COVID-19) ${ }^{6}$, cujos principais sintomas e sinais clínicos são: febre, tosse, dispneia, mialgia, fadiga, dor de cabeça, falta de ar, anosmia e disgeusia, sendo de maior gravidade em pessoas idosas e com comorbidades $^{7}$. A transmissão do SARS-CoV-2 acontece, principalmente, por meio de gotículas de saliva e contato direto com superfícies contaminadas $^{8}$ e, sabe-se que, indivíduos assintomáticos também podem transmitir a doença9 .

No Brasil, foram adotadas diversas estratégias para enfrentamento da COVID-19, incluindo a publicação de protocolos de prevenção e tratamento e o acompanhamento e controle de dados epidemiológicos ${ }^{10}$. No Estado do Paraná, por meio do Decreto n4230 de 16 de março de 2020, foram definidas as medidas para enfrentamento da emergência de saúde pública decorrente do Coronavírus, incluindo a quarentena, isolamento, exames médicos, testes laboratoriais, tratamentos médicos específicos, teletrabalho aos servidores públicos, dentre outras ${ }^{11}$. Diante deste contexto, na mesma data, a Universidade Federal do Paraná (UFPR) suspendeu os calendários acadêmicos dos cursos de graduação, pós-graduação e de educação profissional e tecnológica por 14 dias $^{12}$, sendo que posteriormente esta suspensão foi prorrogada.

Em resposta a crise sanitária e social estabelecida pela pandemia, a Fundação Araucária de Apoio ao Desenvolvimento Científico e Tecnológico do Estado do Paraná, em parceria com a Superintendência Geral de Ciência, Tecnologia e Ensino Superior e a Secretaria de Estado da Saúde do Paraná, propôs, no dia 19 de março de 2020, o Programa de Apoio Institucional para Ações de Prevenção e Cuidados diante da Pandemia do Novo Coronavírus por meio da Chamada Pública 09/2020 para o desenvolvimento de projetos extensionistas por Instituições Estaduais de Ensino 
Superior do Estado do Paraná e pela UFPR ${ }^{13}$.

Deste modo, foi implementado o Programa de Apoio Institucional para Ações Extensionistas de Prevenção, Cuidados e Combate à Pandemia do Novo Coronavírus na atenção às divisas rodoviárias do Estado do Paraná ${ }^{14,15}$. A equipe que atuou no desenvolvimento das ações do projeto foi composta por profissionais e acadêmicos de diversas instituições que se inscreveram em editais específicos para participar como bolsistas, formando uma equipe multiprofissional composta pelas áreas de Enfermagem, Medicina, Medicina Veterinária, Psicologia e Odontologia. Desde o início, o referido programa contou com cirurgiõesdentistas e acadêmicos de Odontologia em sua equipe $^{14}$. Deste modo, o objetivo desse estudo consistiu em relatar a percepção dos discentes de Odontologia quanto sua participação nas ações extensionistas interprofissionais no enfrentamento da pandemia de COVID-19.

\section{MÉTODOS}

Este estudo qualitativo, transversal e descritivo, aborda a percepção dos estudantes de Odontologia relacionada a sua participação no Projeto de Extensão Universitária por meio da atenção às divisas rodoviárias do Estado do Paraná e/ou da atuação como atendentes em serviço de telessaúde para enfrentamento da COVID-19 (Central de Informações COVID-19) e/ou na Farmácia Especial do Estado do Paraná. O referido projeto teve início em 31 de março e foi findado em 30 de dezembro de 2020.

A pesquisa qualitativa busca a compreensão dos fenômenos a partir da perspectiva dos sujeitos ${ }^{16}$. Deste modo, apresenta como matériaprima, a experiência, a vivência, o senso comum e a ação ${ }^{17}$, a fim de compreender as motivações, os significados e os valores que sustentam as opiniões das pessoas acerca de determinado tema ${ }^{18}$.

Para a condução deste estudo a coleta de dados se deu por meio de roteiro com perguntas abertas, enviadas por questionário online via endereço eletrônico, sobre as percepções dos estudantes acerca das contribuições da atuação na Central de Informações COVID-19 e/ou nas divisas rodoviárias para a sua formação.

Durante a pandemia, as entrevistas online se revelaram uma importante alternativa para a condução de pesquisas, proporcionando maior segurança ao pesquisador e aos entrevistados, na medida em que atende à medida de distanciamento social imposto pela COVID-19 ${ }^{19}$.

A amostra foi definida por conveniência, tendo sido incluídos na pesquisa os indivíduos que concordaram em participar por meio da assinatura do Termo de Consentimento Livre e Esclarecido (TCLE). O questionário no Google Forms foi disponibilizado em dezembro de 2020, com intervalo de 7 dias para o seu preenchimento por parte dos estudantes. Nele constavam, as seguintes perguntas: 1) Você participou da ação de atenção as divisas rodoviárias no enfrentamento do COVID-19? 2) Você participou da ação de atenção à regional de saúde (telessaúde ou vigilância em saúde)? 3) Descreva como a atuação dessa ação contribuiu com sua formação em saúde.

Os estudantes selecionados estavam nos dois últimos anos do curso de Odontologia e recebiam bolsas para o desenvolvimento das atividades no Projeto de Extensão Universitária vinculado a Instituições Estaduais de Ensino Superior e à UFPR. Os acadêmicos participantes atuaram no mínimo por 6 meses e no máximo por 9 meses no projeto, com carga horária de 30 horas semanais e bolsa de extensão financiada pela Fundação Araucária de Apoio ao Desenvolvimento Científico e Tecnológico do Estado do Paraná. No período, os estudantes estavam sem atividades didáticas presenciais e remotas na UFPR, e atuaram como bolsistas da Universidade Estadual de Ponta Grossa (UEPG).

A análise dos relatos obtidos se deu por meio da técnica do Discurso do Sujeito Coletivo (DSC). 
Esta técnica fundamenta-se na premissa de que indivíduos convivendo em sociedade compartilham ideias, valores, representações, sentidos, conteúdo dos sentidos e argumentos. Propõe, então, a organização dos depoimentos verbais, por meio das figuras metodológicas, que possibilitam a construção dos $\mathrm{DSCs}^{20}$.

Para essa construção são necessárias quatro operações sobre os depoimentos: as ExpressõesChaves (E-CH), trechos do material verbal dos depoimentos individuais que melhor identificam o seu conteúdo; as Ideias Centrais (ICs), nomes ou expressões que descrevem os sentidos dos depoimentos de cada resposta e também dos conjuntos de respostas de diferentes pessoas, porém com sentidos semelhantes; as Ancoragens (Acs), expressões sintéticas que descrevem as ideologias e valores presentes no material verbal; $\mathrm{e}$ o Discurso do Sujeito Coletivo (DSC), o qual é composto a partir da união das E-CH presentes nos depoimentos, que tem ICs de sentido semelhante, para formar frases encadeadas ${ }^{21-23}$. Por meio do DSC, há a reconstituição de um ser coletivo, opinante sob a forma de um sujeito de discurso apresentado na primeira pessoa do singular ${ }^{24}$.

O presente estudo foi aprovado pelo Comitê de Ética em Pesquisa da Universidade Estadual de Ponta Grossa sob parecer número 32121120.3.0000.0105.

\section{RESULTADOS}

Dentre os estudantes de Odontologia participantes do projeto de extensão, todos os cinco aceitaram responder a essa pesquisa. Destes, quatro relataram ter participado na ação de atenção às divisas rodoviárias e na Central de Informações COVID-19; sendo que um participou apenas das ações na Central de Informações COVID-19; e um atuou também na Farmácia Especial do Estado do Paraná.

Nos quadros 1, 2 e 3 encontram-se descritas as ideias centrais que emergiram a partir da análise dos dados e suas respectivas frequências, bem como os trechos do DSC referentes a cada ideia central. O quadro 1 aborda as ideias centrais referentes às contribuições da ação de atenção às divisas rodoviárias para a formação dos estudantes, as quais envolveram o aprendizado sobre a prevenção da COVID-19 e outros agravos, a oportunidade de atuação interprofissional e a aplicação prática dos conhecimentos teóricos.

Quanto às contribuições da atuação no serviço de Call Center (quadro 2), para a formação dos estudantes, destaca-se a análise de dados epidemiológicos, a oportunidade de atuação interprofissional, o desenvolvimento de habilidades de comunicação e a experiência com telessaúde, a qual poderá ser ampliada futuramente.

Por fim, quanto às contribuições da atuação na Farmácia Especial do Estado do Paraná, destacaram-se o aprendizado acerca dos fluxos de trabalho e da humanização do atendimento, conforme observado no quadro 3.

\section{DISCUSSÃO}

A Extensão vem sendo estimulada pelas comunidades acadêmicas, que reforçam a importância do relacionamento com a comunidade extramuros, por medidas legais, por meio da creditação da extensão nos cursos de graduação, e pelas demandas da própria sociedade ${ }^{25}$. Os achados deste estudo, referentes à contribuição da extensão para o aprendizado, possibilitando a aplicação prática dos conhecimentos teóricos e a atuação interprofissional, o desenvolvimento de habilidades de comunicação e de humanização vão ao encontro das diretrizes da extensão apresentadas na Resolução $n^{\circ} 7$ de 18 de dezembro de $2018^{4}$, no que se refere à interação da comunidade acadêmica com a sociedade por meio do contato com as questões complexas contemporânea; à formação cidadã dos estudantes, pela vivência dos seus conhecimentos de modo interprofissional e interdisciplinar; à formação integral do estudante; e 
à contribuição ao enfrentamento das questões da sociedade brasileira.

O Programa de Apoio Institucional para Ações Extensionistas de Prevenção, Cuidados e Combate à Pandemia do Novo Coronavírus Atenção às divisas rodoviárias do Estado do Paraná proporcionou aos estudantes a experiência de atuação interprofissional, na medida em que incluiu estudantes de diversas áreas, como Enfermagem, Medicina, Medicina Veterinária, Psicologia e Odontologia. Esta experiência foi ressaltada pelos participantes como importante contribuição para sua formação, tanto por aqueles que atuaram na Central de Informações COVID-19, como pelos que atuaram na Atenção às Divisas.

Quadro 1. Ideias centrais referentes às contribuições da ação de atenção às divisas rodoviárias para a formação discente, frequências e discurso do sujeito coletivo

\begin{tabular}{|c|c|}
\hline & Discurso do Sujeito Coletivo (DSC) \\
\hline $\begin{array}{l}\text { A atuação como } \\
\text { fonte de } \\
\text { aprendizado sobre } \\
\text { a prevenção a } \\
\text { Covid-19 e outros } \\
\text { agravos / } 2\end{array}$ & $\begin{array}{l}\text { A atuação contribuiu para o aprendizado sobre a pandemia e a Covid-19, } \\
\text { entender a importância da educação em saúde. O projeto garantiu a } \\
\text { promoção e prevenção não apenas para covid, abordou outras doenças que } \\
\text { aquela população poderia estar suscetível. Me fez entender na prática que a } \\
\text { prevenção de determinadas doenças pode diminuir muito a sobrecarga do } \\
\text { sistema de saúde, além de ser mais rentável. Me fez continuar estudando e } \\
\text { me atualizando sobre o vírus. }\end{array}$ \\
\hline $\begin{array}{l}\text { A oportunidade de } \\
\text { atuação } \\
\text { interprofissional / } 3\end{array}$ & $\begin{array}{l}\text { Através deste projeto, considero que minha formação foi mais ampla, } \\
\text { principalmente levando em consideração a experiência multiprofissional. } \\
\text { [...] Me ajudou a entender a importância de outras profissões da área da } \\
\text { saúde, e na relevância no trabalho em equipe. [...] Proporcionou para mim } \\
\text { (como acadêmica) um melhor conhecimento das particularidades de cada } \\
\text { profissão, e, como em conjunto, todas agregam de forma única para que a } \\
\text { atenção a saúde seja entregue ao "pacientelpopulação" da melhor maneira } \\
\text { que se pode ser oferecida. }\end{array}$ \\
\hline $\begin{array}{r}\text { A atuação } \\
\text { forma de ap } \\
\text { prática } \\
\text { conhecim } \\
\text { teóricos }\end{array}$ & $\begin{array}{l}\text { Muitas vezes na academia somos postos frente a uma formação que não } \\
\text { condiz com a realidade dos serviços de saúde, nisso, projetos como esse nos } \\
\text { transformam e nos mostram a verdadeira vivência nesses espaços, sem falar } \\
\text { no fortalecimento que traz ao Sistema Único de Saúde (SUS) uma vez que } \\
\text { prepara de uma forma mais ampla o profissional. Foi uma experiência com } \\
\text { certeza maravilhosa, que nunca vou esquecer, ainda mais sendo num } \\
\text { período de tanta fragilidade e de história no SUS. Além da oportunidade } \\
\text { prática que obtivemos de desenvolver habilidades de anamnese e } \\
\text { diagnóstico que nos foi proporcionado através das atividades de ronda, } \\
\text { ambulatório móvel (container) e Saúde na Estrada. Também em mente, } \\
\text { posso citar a oportunidade de crescimento pessoal e profissional obtido } \\
\text { durante os quatro meses de atividades realizadas nas Divisas do estado do } \\
\text { Paraná. }\end{array}$ \\
\hline
\end{tabular}


Quadro 2. Ideias centrais referentes às contribuições da atuação na Central de Informações Covid-19 para a formação discente, frequências e discurso do sujeito coletivo

\begin{tabular}{|c|c|}
\hline $\begin{array}{c}\text { Ideia Central/ } \\
\text { Frequência }\end{array}$ & Discurso do Sujeito Coletivo (DSC) \\
\hline $\begin{array}{l}\text { A atuação como fonte } \\
\text { de dados } \\
\text { epidemiológicos / } 1\end{array}$ & $\begin{array}{l}\text { Atuei no controle de óbitos por COVID-19 na segunda regional de } \\
\text { Curitiba, monitorando as mortes, checando fichas prontuários e laudos. } \\
\text { Contabilizando os dados e fazendo o contato com municípios e o estado. } \\
\text { Minha participação foi receber os óbitos de municípios, hospitais e } \\
\text { obituários, checar se estava preenchido corretamente em todas as } \\
\text { plataformas necessárias e se o paciente possuía laudo positivo para } \\
\text { Covid-19. A partir da organização desses dados em uma planilha, era } \\
\text { então enviado diariamente a contagem dos óbitos para o estado e } \\
\text { municípios, contribuindo com o boletim epidemiológico diário. }\end{array}$ \\
\hline $\begin{array}{l}\text { A oportunidade de } \\
\quad \text { atuação } \\
\text { interprofissional / } 3\end{array}$ & $\begin{array}{l}\text { Tive a oportunidade de trabalhar com outras profissões, trocando } \\
\text { conhecimentos e aprendendo mais a cada dia. [...] durante as } \\
\text { teleconsultas orientávamos os pacientes quanto a dieta, afastamento e } \\
\text { medidas preventivas, sendo que cada profissão da saúde teve a } \\
\text { oportunidade de contribuir para a compactação de orientações. A } \\
\text { dinâmica de trabalhar com a equipe multiprofissional é enriquecedora, } \\
\text { pois são adquiridos diversos conhecimentos, compartilhamentos de } \\
\text { experiências com o objetivo de fazer o melhor para os pacientes e toda } \\
\text { sociedade. A importância do trabalho em equipe, reconhecer que cada } \\
\text { pessoa tem o papel importante e fundamental para executar as atividades } \\
\text { e quando realizadas em conjunto torna-se bem elaborado e eficaz. }\end{array}$ \\
\hline $\begin{array}{l}\text { O desenvolvimento das } \\
\text { habilidades de } \\
\text { comunicação / } 3\end{array}$ & $\begin{array}{l}\text { Desenvolvi habilidades de comunicação, as atividades desenvolvidas no } \\
\text { call center me ajudaram a desenvolver principalmente a habilidade de } \\
\text { ouvir e isso auxilia muito no desenvolvimento da produção de uma } \\
\text { anamnese completa, que é primordial em uma "teleconsulta". Como o } \\
\text { projeto foi realizado durante o período de pandemia, ficou mais claro e } \\
\text { evidente a importância de defender a ciência, propagar informações e } \\
\text { conhecimentos a população sobre os hábitos para evitar a propagaçâo } \\
\text { do Coronavírus com linguagem acessível e objetiva. Trabalhar com o } \\
\text { atendimento telefônico, me permitiu ampliar uma dicção e desenvoltura } \\
\text { para comunicação, além disso, fortaleceu no quesito ouvir, pois muitas } \\
\text { vezes como profissionais de saúde somos bons para falar e esquecemos } \\
\text { que ouvir também é enriquecedor, é saber praticar a empatia, colocar-se } \\
\text { no lugar do paciente e ouvi-lo o que tem a dizer, onde muitas vezes, } \\
\text { soluções são encontradas quando sabemos ouvir o outro. }\end{array}$ \\
\hline $\begin{array}{l}\text { Oportunidades de } \\
\text { acompanhar } \\
\text { teleconsultas / } 1\end{array}$ & $\begin{array}{l}\text { Pude acompanhar consultas por telemedicina, que, levando em } \\
\text { consideração o atual cenário, pode ser uma alternativa viável, segura e } \\
\text { moderna para os próximos anos. }\end{array}$ \\
\hline
\end{tabular}


Quadro 3. Ideias centrais referentes às contribuições da atuação na Farmácia Especial do Estado do Paraná para a formação discente, frequências e discurso do sujeito coletivo

\begin{tabular}{|c|c|}
\hline $\begin{array}{c}\text { Ideia Central/ } \\
\text { Frequência }\end{array}$ & Discurso do Sujeito Coletivo (DSC) \\
\hline $\begin{array}{l}\text { A atuação como fonte } \\
\text { de aprendizado dos } \\
\text { fluxos da Farmácia } \\
\text { Especial do Estado do } \\
\text { Paraná / } 1\end{array}$ & $\begin{array}{l}\text { A atuação realizada na Farmácia Especial do Estado do Paraná } \\
\text { localizada na } 2 \text { a regional, foi de suma importância para o aprendizado } \\
\text { pessoal e o profissional. Faltam espaços para descrever o quanto esse } \\
\text { projeto agregou em minha vida. Pois, pude obter novos conhecimentos } \\
\text { sobre o fluxo da maior Farmácia do Estado do Paraná que pertence ao } \\
\text { SUS, sistema a qual eu luto defendo e pude vivenciar a realidade da } \\
\text { prática os princípios que o regem como Integralidade, Equidade e } \\
\text { Universalidade. Percebi através da linha de produção do Entrega em } \\
\text { Casa, a qual os medicamentos são retirados do estoque, são colocados } \\
\text { em caixa, de acordo com a receita médica e ao final do procedimento é } \\
\text { feito a conferência pela farmacêutica responsável, a qual autoriza para } \\
\text { o envio aos pacientes, com isso é perceptível que como profissional de } \\
\text { saúde, a dinâmica do trabalho em equipe onde requer agilidade, } \\
\text { precisão, colaboração e responsabilidade para o melhor desempenho } \\
\text { de todos. }\end{array}$ \\
\hline $\begin{array}{l}\text { O reconhecimento da } \\
\text { importância da } \\
\text { humanização do } \\
\text { atendimento / } 1\end{array}$ & $\begin{array}{l}\text { O atendimento ao público externo para retirada dos medicamentos, } \\
\text { permitiu vivenciar a humanização do cuidado, acolher o paciente e sua } \\
\text { família, entender e compreender a necessidade desses, respeitar suas } \\
\text { individualidades, cada qual está inserido em um contexto social, pois } \\
\text { muitos utilizam medicamentos de uso contínuo, outros com valores } \\
\text { elevados e até os pacientes que recebem medicamentos judicializados } \\
\text { Enfim, as práticas realizadas ampliaram os conhecimentos aprendidos } \\
\text { com Professores que nos inspiram e enriquecem com o trabalho } \\
\text { oportunizado, com isso é possível tornar-se uma futura profissional da } \\
\text { saúde sob um olhar dinâmico pois, pude perceber a relevância das } \\
\text { palavras de Empatia, cooperação (com trabalho em equipe) e } \\
\text { competência, sendo esses os pilares para construção de um } \\
\text { profissionalismo com maior ética e humanidade, sob um novo olhar } \\
\text { promissor pelo SUS em conjunto com a experiência de poder realizar o } \\
\text { trabalho em equipe multiprofissional, exercer com maestria a arte do } \\
\text { cuidado e sempre zelar pela qualidade no atendimento aos pacientes }\end{array}$ \\
\hline
\end{tabular}

De acordo com a Organização Mundial da Saúde $^{26}$, a educação interprofissional ocorre quando estudantes de duas ou mais áreas profissionais aprendem sobre os outros, com os outros e entre si para a prática colaborativa e melhoria dos resultados em saúde. Frente ao cenário atual da pandemia de coronavírus, o trabalho interprofissional, articulando ações colaborativas, para potencialização da capacidade de solucionar problemas, tornou-se ainda mais 
necessário para maximizar os resultados obtidos, evidenciando a necessidade de formação de profissionais de saúde aptos para esta atuação ${ }^{27}$.

Outro ponto destacado pelos estudantes foi a atuação na extensão como fonte de aprendizados e aplicação dos conhecimentos. Um estudo conduzido por Fadel e colaboradores ${ }^{28}$, avaliando o impacto exercido pela vivência dos estudantes em atividades extensionistas, também identificou o desenvolvimento de habilidades profissionais $\mathrm{e}$ pessoais como importante ponto. No referido estudo constatou-se que os estudantes percebem, na atividade extensionista formas de romper com o enfoque tradicional do ensino, promovendo maior convivência, troca de conhecimentos, trabalho em equipe e uma resposta mais satisfatória às necessidades em saúde da população ${ }^{28}$. Além disso, as ações de extensão trazem impacto positivo para a comunidade, na medida em que proporcionam aprendizado, mudanças de hábitos, promoção de saúde e prevenção de doenças, refletindo também na formação do futuro profissional $^{29}$.

Moimaz e colaboradores ${ }^{30}$, em resultado de pesquisa conduzida com estudantes do curso de Odontologia participantes de um projeto de extensão, também constataram como principais impactos o recebimento de estímulos para busca de novos conhecimentos e a fixação do conteúdo teórico na prática; o aprimoramento do conhecimento técnico-científico e prático para a atenção humanizada; e o trabalho em equipe.

Outro aspecto mencionado pelos estudantes refere-se à telessaúde e a forma como poderá ser empregada, inclusive futuramente, em cenário póspandemia. O uso de tecnologias de telessaúde para atendimento pré-clínico não presencial, realização de agendamento, avaliação dos sintomas, canais de chat e canais telefônicos ampliaram-se no contexto pandêmico atual ${ }^{31}$. A aplicação efetiva da telessaúde para enfrentamento ao coronavírus poderá ampliar a sua aceitação para outras áreas da saúde no futuro, incluindo o acompanhamento de doenças crônicas ${ }^{32}$.

Em se tratando especificamente da teleodontologia, esta é uma importante ferramenta para auxílio às atividades odontológicas por meio da teleassistência e da teleducação. É importante destacar que a teleassistência não substitui as consultas presenciais, sendo recomendada para casos específicos, em que o paciente possui bom entendimento e colaboração para a realização dos cuidados em saúde bucal em sua residência, em conformidade com a orientação recebida dos profissionais $^{33}$.

Em 2020, o Conselho Federal de Odontologia publicou a Resolução CFO-226, de 04 de junho, a qual regulamenta o exercício da Odontologia a distância, mediado por tecnologias. A referida Resolução veta o seu exercício para fins de consulta, diagnóstico, prescrição e elaboração de plano de tratamento odontológico; admite o telemonitoramento, por meio do acompanhamento a distância dos pacientes que estejam em tratamento, no período entre as consultas; e admite durante o período de calamidade pública a teleorientação realizada por cirurgião-dentista com o objetivo de identificar, por meio de aplicação de questionário pré-clínico, o melhor momento para a realização do atendimento presencial ${ }^{34}$.

A extensão universitária traz importantes contribuições para a formação de cirurgiõesdentistas, como a ampla compreensão do processo saúde doença, o desenvolvimento de habilidades práticas, a ampliação da resolutividade, e o atendimento às necessidades da comunidade de forma integral ${ }^{35}$. Os resultados obtidos na presente pesquisa reforçam a importância da extensão, no sentido de formar cidadãos e profissionais comprometidos com a promoção de saúde, com a resolutividade dos serviços de saúde, a integralidade da atenção e com a realidade sanitária e social do país. 


\section{CONCLUSÃO}

A percepção dos estudantes participantes deste estudo evidenciou como principais contribuições do Projeto de Extensão para a sua formação a obtenção de novos conhecimentos, a oportunidade de atuação interprofissional, a aplicação prática dos conhecimentos teóricos, proporcionando experiência profissional, o aprendizado técnico-científico, a promoção de acolhimento, da escuta qualificada e de atendimento humanizado. Deste modo, percebe-se que está atendendo às diretrizes para a Extensão na Educação Superior Brasileira, promovendo a formação integral e cidadã dos estudantes, propiciando a vivência dos seus conhecimentos, de modo interprofissional e interdisciplinar.

Destaca-se também o papel da Odontologia, como área da saúde, que junto com as demais profissões da saúde, não teria como não estar envolvida nesse momento de emergência em saúde pública, como a pandemia de COVID-19. A atuação dos estudantes no enfrentamento do COVID-19, foi fundamental para demonstrar a eles a importância de um sistema público universal, como o Sistema Único de Saúde, bem como que o processo de trabalho da Odontologia não se limita somente a prática clínica bucal. O trabalho interprofissional presente no projeto aqui relatado, demonstra o papel essencial de todos os profissionais e estudantes da saúde na atenção à saúde da população no enfrentamento do COVID19.

\section{ABSTRACT \\ Dental students in the fight against COVID-19: student's perception of an interprofessional extension project}

This paper reports the perception of Dentistry students concerning their participation in interprofessional extension actions in the management of the COVID-19 pandemic. For that purpose, a qualitative, cross-sectional and descriptive study was performed, using a script with open questions and a self-applied structured questionnaire sent to students participating in the University Extension Project by attention to the road borders of the State of Paraná and/or as attendants in the telehealth service in the management of COVID-19 (COVID-19 Information Center) and/or the Special Pharmacy of the State of Paraná. The reports were analyzed by the Collective Subject Discourse. All Dentistry students who participated in the project agreed to respond to this survey. The main contributions of the Extension Project to their training were identified as acquisition of new knowledge, opportunity for interprofessional action, practical application of theoretical knowledge, technical-scientific learning, promotion of reception, qualified listening and humanized care. Thus, it is concluded that the guidelines for Extension in Brazilian Higher Education are being met, promoting the integral and citizen training of students, providing the experience of their knowledge in an interprofessional and interdisciplinary manner.

Descriptors: Coronavirus Infections. Dentistry. Community-Institution Relations. Education.

\section{REFERÊNCIAS}

1. Brasil. Resolução $n^{\circ} 3$, de 21 de junho de 2021. Institui as Diretrizes Curriculares Nacionais do curso de graduação em Odontologia e dá outras providências. Diário Oficial da União, Brasília, DF, 22 jun.2021, Seção 1, p. 77.

2. Costa I CC, Unfer B, Oliveira AGRC, Arcieri RM, Saliba NA, Mimaz S. Integração Universidade-comunidade: análise das atividades extra-murais em Odontologia nas Universidades brasileiras. Rev Cons Reg Odontol. 2000; 3(6):146-53.

3. Moraes SLDD, Tamaki R, Sobral APV, Júnior JFS, Leão RDS, Silva BGD, Gomes J MDL. Impacto de uma experiência extensionista na formação universitária. Rev Cir Traumatol Buco-maxilo-fac 2016; 16(1):39-44. 
4. Brasil. Resolução $\mathrm{n}^{\circ} 7$, de 18 de dezembro de 2018. Estabelece as Diretrizes para a Extensão na Educação Superior Brasileira e regimenta o disposto na Meta 12.7 da Lei $n^{\circ}$ 13.005/2014, que aprova o Plano Nacional de Educação - PNE 2014-2024 e dá outras providências. Diário Oficial da União, Brasília, DF, 19 dez. 2018, Seção 1, p. 49.

5. Brasil. Ministério da Saúde. Política Nacional de Promoção da Saúde. 3.ed. Brasília: MS; 2010.

6. Zhu N, Zhang D, Wang W, Li X, Yang B, Song $\mathrm{J}$, et al. A novel coronavirus from patients with pneumonia in China, 2019. N Engl J Med. 2020; 382(8):727-33.

7. Rodriguez-Morales AJ, Cardona Ospina JA, Gutiérrez-Ocampo E, Villamizar-Peña R, Holguin-Rivera Y, Escalera-Antezana JP, et al. Clinical, laboratory and imaging features of COVID-19: a systematic review and meta-analysis. Travel Med Infect Dis. 2020;34:101623.

8. Caldas JP, Tavares M. Epidemiologia da COVID-19. Da emergência um novo vírus humano à disseminação global de uma nova doença - Doença por Coronavírus 2019 [Internet]. 2020 [Acesso em 24 mar. 2021]. Disponível em: http://asset.youoncdn.com/ ab296ab30c207ac641882479782c6c34/070 b44658f5569888804a14826ae273c.pdf.

9. Singhal T. A review of coronavirus disease2019 (COVID-19). Indian J Pediatr. 2020;87(4):281-6.

10. Silva ACR, de Jesus TS, Santos SS, Santos GJ, Rodrigues WP. Covid-19, o novo coronavírus: um alerta emergencial para as principais estratégias de prevenção da saúde pública. Scire Salutis. 2020;10(2):26-34.

11. Paraná. Decreto n 4230 de 16 de março de 2020. Dispõe sobre as medidas para enfrentamento da emergência de saúde pública de importância internacional decorrente do Coronavírus - COVID-19. [Acesso em 11 nov. 2021]. Disponível em: https://www.jusbrasil.com.br/diarios/28781 9206/doepr-caderno-normal-executivo-1603-2020-pg-3.

11. Universidade Federal do Paraná. Despacho $n^{\circ}$ 38/2020/UFPR/R/GAB/SOC. Suspensão dos Calendários Acadêmicos da UFPR. [Acesso em 11 nov. 2021]. Disponível em: http://www.soc.ufpr.br/portal/wp-content/u ploads/2020/03/Ad-Referendum-Suspens\% C3\%A3o-dos-Calend\%C3\%A1rios-Acad\% C3\%AAmicos-da-UFPR.pdf.

12. Fundação Araucária. Chamada Pública 09/2020. Ação de Extensão Contra o Novo Coronavírus. Programa de Apoio Institucional para Ações Extensionistas de Prevenção, Cuidados e Combate à Pandemia do Novo Coronavirus. [Acesso em 11 nov. 2021]. Disponível em: http://www.seti. pr.gov.br/sites/default/arquivos_restritos/fil es/documento/2020-03/chama_publica_co mbate ao_coronavirus.pdf.

13. Silva ROC, Bonan KFZ, Rosa FC, Cunha GR, Müller EV, Favero GM, et al. Ações

14. extensionistas na prevenção, cuidados e combate à pandemia por Coronavírus nas divisas do Paraná. J Nurs Health. 2020;10 :e20104025.

15. Perez J, Schonrock J, Cardoso LS, Silva MN, Oliveira NR, Gomes SR, et al. Central de Informações Covid-19 como ação de enfrentamento ao novo coronavírus: Relato de experiência. Rev Saúde Desenv Hum. 2021; 9(3):1-12.

16. Godoy AS. Introdução à Pesquisa Qualitativa e suas Possibilidades. Rev Admin Empresas. 1995; 35(2):57-63.

17. Minayo MCS. Análise Qualitativa: teoria, passos e fidedignidade. Ciênc Saúde Colet. 2012;17(3): 621-6.

18. Fraser MTD, Gondim SMG. Da fala do 
outro ao texto negociado: Discussões sobre a entrevista na pesquisa qualitativa. Paidéia. 2004; 14(28):139-52.

19. Schmidt B, Palazzi A, Piccinini CA. Entrevistas online: potencialidades e desafios para coleta de dados no contexto da pandemia de COVID-19. Rev Fam Ciclos Vida Saúde Contexto Soc. 2020; 8(4), 9606.

20. Lefèvre F, Lefèvre AMC. O pensamento coletivo como soma qualitativa. Faculdade de Saúde Pública / USP, São Paulo; 2003. [Acesso em 14 jul. 2021]. Disponível em: http://www.fsp.usp.br/qualisaude/soma\%20 qualitativa $\% 209 \% 20 \mathrm{de} \% 20 \mathrm{fevereiro} \% 20 \mathrm{de}$ $\% 202004 . \mathrm{htm}$.

21. Lefèvre F, Lefèvre AMC. Depoimentos e Discursos: uma proposta de análise em pesquisa social. Brasília: Líber Livro Editora; 2005.

22. Lefèvre F, Lefèvre AMC. O discurso do sujeito coletivo: um novo enfoque em pesquisa qualitativa (desdobramentos). Caxias do Sul: EDUSC; 2005.

23. Lefèvre F, Lefèvre AMC. Pesquisa de Representação Social: Um enfoque Qualiquantitativo. 2. ed. Brasília: Liber Livro Editora; 2012.

24. Lefèvre F, Lefèvre AMC. O Sujeito Coletivo que fala. Interface Comun Saúde Educ. 2006; 10(20): 517-24.

25. Silva WP. Extensão universitária: um conceito em construção. Extensão \& Sociedade. 2020; 11(2):21-32.

26. World Health Organization. Framework for action on interprofessional education \& collaborative practice. World Health Organization: Geneva; 2010.

27. Souza, SVD, Rossit, RAS. Dilemas e Perspectivas dos recursos humanos em saúde no contexto da pandemia. Enferm Foco. 2020; 11(1):68-73.
28. Fadel CB, Bordin D, Kuhn E, Martins LD. O impacto da extensão universitária sobre a formação acadêmica em Odontologia. Interface Comun Saúde Educ. 2013; 17(47): 937-46.

29. Souza KC, da Costa LP, Barreto NMF, Siqueira AAMG, de Souza SS, Rocha ES, et al. Políticas públicas e educação em saúde nos projetos de extensão na universidade. Res Soc Devel. 2021; 10(4), e58010414379.

30. Moimaz SAS, Bordin D, Gomes AM de P, Fadel CB, Garbin CAS, Saliba NA. Extensão universitária na ótica de acadêmicos: o agente fomentador das Diretrizes Curriculares Nacionais. Rev ABENO. 2015; 15(4): 45-54.

31. Celuppi IC, Lima GDS, Rossi E, Wazlawick RS, Dalmarco EM. Uma análise sobre o desenvolvimento de tecnologias digitais em saúde para o enfrentamento da COVID-19 no Brasil e no mundo. Cad Saúde Públ. 2021; 37(3):e00243220.

32. Caetano R, Silva AB, Guedes ACCM, Paiva CCND, Ribeiro GDR, Santos DL, Silva RMD. Desafios e oportunidades para telessaúde em tempos da pandemia pela COVID-19: uma reflexão sobre os espaços e iniciativas no contexto brasileiro. Cad Saúde Públ. 2020; 36, e00088920.

33. Nascimento ADA, de Holanda LAL, Borges BS, Pereira FT, Frade AL, de Barros Santos C. de Lacerda Vidal AK. Teleodontologia, Formação Profissional e Assistência Odontológica no Sistema Único de Saúde Brasileiro: Relato de Experiência. J Health. 2020; 1(1):51-67.

34. Conselho Federal de Odontologia. Resolução CFO-226, de 04 de junho de 2020. Dispõe sobre o exercício da Odontologia a distância, mediado por tecnologias, e dá outras providências. [Acesso em 26 jun. 2021]. Disponível em: 
https://sistemas.cfo.org.br/visualizar/atos/R

ESOLU\%c3\%87\%c3\%830/SEC/2020/226.

35. Pizzolatto G, Dutra MJ, Corralo DJ. A extensão universitária na formação do cirurgião-dentista. Rev ABENO. 2021; 21(1): 974.

\section{Correspondência para:}

Thabata Cristy Zermiani

e-mail: thabata.zermiani@gmail.com

Programa de Pós-graduação em Políticas

Públicas

Setor de Ciências Sociais Aplicadas

Av. Prefeito Lothário Meissner, 632 Térreo

Jardim Botânico

80210-170 Curitiba/PR 